Canadian Journal of Family and Youth, 13(3), 2021, pp. 378-384

ISSN 1718-9748@ University of Alberta

http://ejournals,library,ualberta.ca/index/php/cjfy

\title{
The Effects of Video Games on Children's Well-Being
}

Sarah Salman, MacEwan University

\section{Introduction}

Recognizing the positive aspects of video games and media in children's lives can be identified when parents and educators make inquiries and children's voices are heard. Although the importance of understanding children's perspectives cannot be emphasized enough, researchers are also concerned about the effects of video games and media on children's academic performance, development, and overall health and well-being. Investigating the current research on the impact of video games on children is significant as it reveals issues that arise with a relatively new form of media that has captivated many young audiences. Exploring the nuances of video games that manifest in the variety of gaming styles and considering the age at which children interact provides insight into the relationship between parental involvement and children's behavioral outcomes. Familiarizing oneself with a cross-disciplinary analysis of this matter helps parents and educators understand their roles in developing an approach to guide children. By undertaking the task of assessing the influence of video games and media on children, the public can form an informed opinion and avoid merely labeling new media as inherently harmful. Rather, a cross-disciplinary analysis identifies the potentially detrimental effects of media saturation and reveals the consequences of specific mediation approaches while perhaps enabling parents to address their concerns yet set expectations and guidelines grounded in mutual understanding. It is hypothesized that video games and parenting styles may have a synergistic effect to impact childhood development either negatively or positively.

\section{Literature Review}

The existing research on the effect of video games and media on children suggests that specific harms are associated with these mediums. However, it is worth considering how children are affected by advertising and marketing as a foundational basis of identifying initial and underlying issues. According to Calvert (2008), advertising and marketing towards children is problematic as it utilizes approaches such as product placement online and in films and video games. The issue with product placement is that younger children below the age of eight are particularly impressionable and unable to critically examine media messages and persuasive intent due to underdeveloped cognitive skills. In addition, Calvert (2008) states that older children may also be unable to recognize advertising's persuasive intent within newer marketing techniques 
found in commercial and program content. The effects of marketing on children, who often are heavy media users, are far-reaching as children have access to various devices and platforms, including online video games.

Through various marketing techniques, marketers can integrate subtle or overt commercial messages within online video games utilizing endorsements, brands, and audiovisual mechanisms to increase attention, familiarity, and consumer loyalty (Calvert, 2008, p. 209). This illustrates that developing videogames is a practice primarily concerned with leveraging consumer interest and maintaining an appeal for videogames, thereby raising concerns regarding the adverse effects on children. When corporate interests precede children's safety and are coupled with data collection and tracking minors' activities to gain knowledge about consumer behaviour, the privacy of children becomes a concern. Accordingly, advertising that drives young consumers' unconscious needs and emotions and capitalizes on their information is an area of great concern given that young children do not have the capacity to comprehend marketing intent. Calvert (2008) emphasizes the benefits of media literacy training for older children, who are more receptive to it, to educate them about deceptive advertising. Although regulatory measures by organizations such as the Federal Trade Commission (FTC) are in place to ensure commercial and product advertising limits, some limitations exist when regulating interactive technology marketing practices found in video games (Calvert, 2008, p. 223).

Considering the discrepancies when regulating marketing practices in video games and the gaming industry's profit-oriented nature, it is not surprising that parents have concerns in attempting to moderate children's usage of media. Gentile et al. (2012) researched parental monitoring of children's media use which involved reports from 1,323 participants, including parents, elementary-aged children, and teachers. These reports identified patterns in setting time limits for video games and television and monitoring methods based on the child's age and parental education and income. The research highlights children's motivations for seeking access to video games during middle childhood, citing avoidant behaviour or the need to escape and form social connections. The authors concentrate on active mediation, the most frequently researched practice of educating children regarding the "technical aspects of media programming," which enhances comprehension, develops critical thinking, and reduces undesirable responses to advertising (Gentile et al., 2012, p. 471). Further, the authors address the positive outcomes of restrictive mediation regarding higher academic success, less aggression, and exposure to stereotypical gender roles. However, the research does not contextualize the findings within the scope of parenting styles and rather attributes differences in parental monitoring to demographic and family characteristics.

Nevertheless, active, and restrictive mediation are both linked to parental education. However, the former is more reflective of families with higher education and income and "involved communication style[s]" while the latter is "the preferred monitoring choice among lowincome parents" (Gentile et al., 2012, p. 474). The authors suggest that both types of parental mediation act as protective measures; however, the full extent of the benefits on children's development is not explored. A strength of the research methodology is the descriptive statistics indicating discrepant responses from both children and parents regarding parental monitoring measures due to social desirability biases. Research by Gentile et al. (2012) also expanded the research study to include the monitoring of video game use - an otherwise disregarded aspect of 
prior analyses.

In a similar research study, Martins et al. (2017) examined parental mediation of video game play through an online survey designed for 433 parents of children aged five to eighteen. The research's main feature was to determine the valence of mediation for three categories: positive, negative, and neutral, to determine the relationship between mediation techniques and parental involvement, and to assess the possible correlation with child delinquency. Similar to the research by Gentile et al. (2012), this study evaluated the effects of active and restrictive mediation and discovered that involved parents were the most likely to utilize each of the mentioned strategies than less involved parents. By comparison, Gentile et al. (2012) concentrated on demographic and family characteristics (such as education level and socioeconomic status) as predictors of preferred mediation strategies. Contrastingly, Martins et al. (2017) examine parental involvement and monitoring of children's video game use and highlight that restrictive and negative mediation is linked with child delinquency. Research by Martins et al. (2017) builds upon previous studies and examines parental mediation techniques and their effects.

In summary, restrictive mediation occurs when parents enforce rules and control over media exposure to set time limits and restrictions for media consumption, which resulted in an increase in childhood delinquency. Positive active mediation involves communication between parents and children to negotiate media consumption choices and is utilized by parents to encourage positive behaviours (i.e., viewing programs perceived to be educational) and discourage risk associated behaviours such as disclosing personal information online. Contrastingly negative active media mediation denotes rejecting media content or voicing disapproval and is linked with lower aggression in children. When co-viewing/using occurs with parents and children, there are greater educational and prosocial benefits and lower risks (Martins et al., 2017, p. 1217). A limitation of prior studies on parental mediation of video games is the lack of differentiation between the three subtypes of active mediation strategies, and the authors' comprehensive investigation regarding effects on children's development is an asset.

Furthermore, in comparison to the study on mediation by Gentile et al. (2012), the authors of this article illustrate that children's age and sex determine the preferred mediation method (Martins et al., 2017, p. 1219). However, the evidence suggests that parental age, sex, and attitude towards video games also predicts the use of parental mediation (Martins et al., 2017, p. 1219). Overall, the findings support that parents are involved in monitoring children's use of video games and other mediums regardless of whether they choose to co-view. However, it is unclear why negative mediation is less effective with middle-aged and older children than younger children (Martins et al., 2017, p. 1231). Revising this section would clarify if family communication patterns or other variables are predictive of child outcomes or if receptivity towards negative mediation is merely representative of age-based differences.

Parental mediation or monitoring of children's video game and media use is of concern for numerous reasons, including its effect on academic performance. In a longitudinal study conducted by Mundy et al. (2020), children aged eight to nine and their parents were recruited to measure children's academic performance at baseline and between ten and eleven years of age. The authors emphasize the significance of media's effect on children during the mid-primary years when interactions with media increase while addressing the lesser-known impacts of new media such as video games. Consequently, the research examines the long-term effects of media on academic 
performance, including measures for videogames with consideration for descriptive factors such as age, sex, and socioeconomic status. The authors measured rates of media use across two points in time in childhood and discovered slight increases for children in grade 3 (where the average age of children was 9.0 years) and in grade 5 (where the average age of children was 10.9 years) (Mundy et al., 2020, p. 6).

Moreover, Mundy et al. (2020) found a minimal relationship between video game use and "grade 3 reading and numeracy scores" (p. 8). Also, playing video games was not correlated with reading or numeracy scores during grade 5. A strength of the authors' methodological approach is the longitudinal study that captures two points in childhood while measuring academic performance with a national achievement test. However, to fully recognize the effects of video games on children's reading and numeracy performance, extending the achievement tests for participants up to fourteen years of age may be required to observe the impact in late childhood. Furthermore, the drawbacks of utilizing self-report measures for participants aged fourteen and up, in place of achievement tests, is that individuals may misrepresent academic data due to social desirability bias or having knowledge about the research objectives. Lastly, expanding the sample size to include cases where parents do not regulate video game playing would implicate the cumulative effects of long-term media use (Mundy et al., 2020, p. 11).

A second longitudinal study conducted by Lobel et al. (2017) examined the effects of video games on children's psychosocial development (p. 884). The study included reports from 194 children between 7.27 to 11.43 years of age regarding gaming frequency, tendency to play violent video games, and cooperative and competitive play. Parents also reported children's psychosocial health, changes, and behaviour. The authors contribute to prior discussions on the positive and negative consequences of gaming and explore the relationship between different forms of video game playing and children's psychosocial development. The authors acknowledge that despite concerns regarding the effects of video games on prosocial behaviour, gaming has become more interactive, and "potential benefits of gaming" are existent (Lobel et al., 2017, p. 885). According to the authors, previous research centralized on internalizing problems such as anxiety and depression within "problematic gamers" - a term that refers to children who seek gaming as a means of escape (Lobel et al., 2017, p. 885). However, the research shows that avoidant behaviours can exacerbate underlying issues, and the exact cause and effect between internalizing problems and gaming is unknown. With regard to hyperactivity and attention as outcomes of gaming, there are conflicting findings, with some studies suggesting a bidirectional effect and others highlighting the cognitive benefits (Lobel et al., 2017, p. 885).

The research supports that cooperative gaming may promote prosocial behaviour and reduce aggressive behavior. Conversely, competitive gaming may be a predictor of aggressive behaviours both in the short-term and long-term (Lobel et al., 2017, p. 886). The authors state that children are particularly susceptible to video game influence as they are undergoing social and emotional development. Lobel et al. (2017) also examine externalizing problems (manifesting as interpersonal conflict or bullying) as an aspect of children's psychosocial health. The authors intended to illustrate gaming behavior differences by comparing gaming patterns, and the results showed that gaming frequency impacted internalizing behaviours although violent gaming was not shown to affect externalizing behaviours or decrease prosocial behaviour. To replicate the research 
findings, this study can be expanded to determine other causes that produce internalizing problems in children with maladaptive behaviours. Furthermore, considering the role of parental mediation is necessary to understand the effects of violent gaming for younger children in the sample (Lobel et al., 2017, p. 893).

Wilson (2008) underscores the interplay between media effects and anti-social behaviour taking form in violence or aggression. The concept of social cognitive theory (the tendency for children to emulate media influences) is a prominent research theme. According to Wilson (2008), experimental evidence suggests that violent video games were influential in children's behaviours towards competitors in computer games, thus "increas[ing] short-term aggression" in both boys and girls (p. 102). One of the research objectives was to identify the relationship between different types of media content and socioemotional development. The findings showed that violent video games have less of an effect on aggressive behaviour than television. However, a limitation of recent research regarding identifying children's aggression is the few studies in this area that mainly involve adults. Thus, the smaller effect of video games on children must be interpreted with caution. Moreover, parents' involvement in monitoring children's media content and establishing time limits while simultaneously encouraging critical media consumption is believed to be essential in increasing prosocial benefits (Wilson, 2008, p. 110). Overall, this research raises concerns that parental influence is a crucial aspect of monitoring children's video game and electronic media use. Similar to the previously discussed studies, the research reiterates that longterm media exposure effects in children are relatively unknown, particularly in the case of newer media such as video games. Hence, more research involving children is required to determine the nature and extent of ramifications.

Vandewater et al. (2004) examined the effects of gaming behaviour on children's activity levels and physical well-being and hypothesized that video games facilitate a sedentary lifestyle contributing to a decrease in physical activity and an increase in caloric intake when product placement occurs. Although the research did not solely concentrate on video games, the prevalence of obesity linked with the frequency of television viewing is believed to have similar effects as with videogames. Children between the ages of 1-12 composed the study's representative sample, and verbal or written accounts (24-h time-use diaries) were utilized to estimate amounts of video game and television use. Vandewater et al. (2004) categorized children's leisure activity participation to understand the relationship "between obesity, media use, and activity level" (p. 75). A strength of the research is the breadth of variables (such as age, gender, ethnicity, and parental education and income) measured as predictors of activity and media use and predictors of weight status. Children who moderately used media had lower weight status than those who used it excessively or for very little. Overall, the results indicate that there is evidence for video games being linked to obesity; however, research on the effects of video games is underdeveloped, and further research is required to determine the causal relationship between video games and obesity (Vandewater et al., 2004, p. 81). To replicate the research procedures, it is necessary to explicate the implications of electronic game play and non-game media use in separate analyses to observe the respective effects on children's weight status and activity levels. Also, considering the amount of access to electronic devices (including tablets, gaming consoles, smartphones, and laptops) as 
a variable predicting an increase in sedentary activities would be valuable. Lastly, including the impact of video games on children with disabilities in future research and the consequences on obesity and activity levels would better inform parents and teachers.

\section{Conclusion}

In summary, the research has shown that concerns for children's well-being are partly due to the fact that the study of newer media, such as video games, is not extensively explored. The cumulative effect video games have on children's development is somewhat unknown; however, it is evident that children are susceptible to marketing persuasion and that parental mediation strategies reinforce positive or undesired behaviours such as delinquency. Also, more research is required on factors such as family communication patterns, children with disabilities, and level of parental involvement to fully capture predictors of child outcomes in the long-term. A stronger emphasis on video games as a leisure activity for children and considering the amount of access to devices and platforms is also advantageous. Including qualitative interviews with children, as part of the research design, may be more reliable than utilizing self-reports as it effectively accesses children's thoughts and feelings and reduces the risk of social desirability bias. Furthermore, extending achievement tests until late childhood is also advantageous in determining the long-term effects of video game use on academic performance. 


\section{References}

Calvert, S. L. (2008). Children as Consumers: Advertising and Marketing. Future of Children, 18(1), 205-234.

Martins N, Matthews N. L., Ratan R. A. (2017). Playing by the Rules: Parental Mediation of Video Game Play. Journal of Family Issues, 38(9), 1215-1238. https://www.doi.org/10.1177/0192513X15613822

Gentile, D., Nathanson, A., Rasmussen, E., Reimer, R., \& Walsh, D. (2012). Do You See What I See? Parent and Child Reports of Parental Monitoring of Media. Family Relations, 61(3), 470-487. https://www.jstor.org/stable/41495223

Lobel, A., Engels, R. C. M. E., Stone, L. L., Burk, W. J., \& Granic, I. (2017). Video Gaming and Children's Psychosocial Well-being: A Longitudinal Study. Journal of Youth and Adolescence, 46(4), 884-897. https://doi.org/10.1007/s10964-017-0646-Z

Mundy, L. K., Canterford, L., Hoq, M., Olds, T., Moreno-Betancur, M., Sawyer, S., Kosola, S., \& Patton, G. C. (2020). Electronic media use and academic performance in late childhood: A longitudinal study. PLOS ONE, 15(9), 1-15. https://doi.org/10.1371/journal.pone.0237908

Vandewater, E. A., Shim, M., \& Caplovitz, A. G. (2004). Linking Obesity and Activity Level with Children's Television and Video Game Use. Journal of Adolescence, 27(1), 71-85.

Wilson, B. (2008). Media and Children's Aggression, Fear, and Altruism. The Future of Children, 18(1), 87-118. https://www.jstor.org/stable/20053121 